\title{
Mechanism of mesenchymal stem cells in spinal cord injury repair through macrophage polarization
}

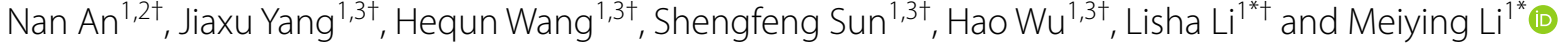

\begin{abstract}
Treatment and rehabilitation of spinal cord injury (SCI) is a major problem in clinical medicine. Modern medicine has achieved minimal progress in improving the functions of injured nerves in patients with $\mathrm{SCl}$, mainly due to the complex pathophysiological changes that present after injury. Inflammatory reactions occurring after $\mathrm{SCl}$ are related to various functions of immune cells over time at different injury sites. Macrophages are important mediators of inflammatory reactions and are divided into two different subtypes (M1 and M2), which play important roles at different times after SCl. Mesenchymal stem cells (MSCs) are characterized by multi-differentiation and immunoregulatory potentials, and different treatments can have different effects on macrophage polarization. MSC transplantation has become a promising method for eliminating nerve injury caused by $\mathrm{SCl}$ and can help repair injured nerve tissues. Therapeutic effects are related to the induced formation of specific immune microenvironments, caused by influencing macrophage polarization, controlling the consequences of secondary injury after $\mathrm{SCl}$, and assisting with function recovery. Herein, we review the mechanisms whereby MSCs affect macrophage-induced specific immune microenvironments, and discuss potential avenues of investigation for improving SCI treatment.
\end{abstract}

Keywords: Spinal cord injury, Inflammatory microenvironment, Macrophage polarization, Mesenchymal stem cells

\section{Background}

Spinal cord injury (SCI) is a serious complication of spine injury, which often leads to serious dysfunction of the limbs below the injured segment. It is one of the most common diseases leading to disability, and no effective treatments have been developed. Secondary injury is initiated shortly after the occurrence of primary SCI, resulting in irreversible damage to neurons and oligodendrocytes in the injured area. Inflammation caused by immune cells, such as macrophages, during the early

\footnotetext{
*Correspondence: lilisha@jlu.edu.cn; limeiying@jlu.edu.cn

${ }^{\dagger}$ Nan An, Jiaxu Yang, Hequn Wang, Shengfeng Sun and Hao Wu contributed equally to this work

${ }^{1}$ The Key Laboratory of Pathobiology, Ministry of Education, Jilin University, 126 Xinmin Street, Changchun 130021, Jilin, People's Republic of China

Full list of author information is available at the end of the article
}

and late stages of SCI is the main cause of secondary injury after SCI and prolongs the process of rehabilitation. In recent years, research on the application of MSCs for treating SCIs has gradually increased. Previous findings have shown that MSCs may elicit definite effects on macrophage polarization. Therefore, during clinical treatment, MSCs are expected to affect macrophage polarization, so as to change the inflammatory microenvironment of SCI and promote the recovery of neural functions.

In this review, we summarize the influence of macrophage polarization on the inflammatory microenvironment during SCI, the therapeutic effects of MSCs on SCI, and the regulatory mechanism of MSCs on macrophage polarization. We hope that the data highlighted in this review may provide guidance for stem cell-based therapy for SCI in the future. 


\section{The pathophysiology of $\mathrm{SCI}$}

SCI leads to motor, sensory, and autonomic nerve damage. In some cases, these defects may be caused by a loss of oligodendrocytes and demyelination in the remaining axons, resulting in slow or blocked conduction throughout the lesion [1]. SCI can be divided into primary and secondary SCI. The former refers to injury caused by the direct or indirect effects of an external force on the spinal cord. The latter refers to spinal cord edema caused by an external force, hematoma caused by small vessel hemorrhage in the spinal canal, compression fractures and ruptures of intervertebral disc tissues, and further damage to the spinal cord due to spinal cord compression. Due to its complex etiology, the pathogenesis of SCI is complex and includes oxidative stress, inflammatory responses, and glial scar formation. A key feature of SCI is that tissue destruction causes secondary damage and non-regressive inflammation, which aggravates the loss of function and impedes recovery. The early onset of inflammation after traumatic SCI emphasizes the importance of acute intervention after the initial trauma [2, 3]. Spinal neurons are sensitive to various injury-inducing factors, such as hypoxia and ischemia. Thus, when SCI interrupts downward projection, neurological dysfunction can result from subsequent denervation of lumbar motor neurons, neuronal cell death, and vascular injury [4-6].

Yet, despite all the damage caused, SCI is not untreatable. Maturation of the human immune system is related to recovery from injury-induced pathology and the recovery of neurological functions after SCI [7]. After the occurrence of SCI, immune cells can repair the tissue, close the wound, promote the removal of debris, inhibit the inflammatory response, and form a dense matrix by mobilizing immune cells and glial cells to form a protective barrier [8]. In addition, several prognostic indicators of SCI have been developed [9]. Among the various mechanisms leading to secondary SCI, inflammation is the most important because it directly or indirectly controls the sequelae after SCI. Inflammation in SCI can be divided into the following stages: neutrophil stimulation and invasion of the resident microglia on the second day, monocyte recruitment into the focus on the third through seventh days, and scar removal by anti-inflammatory macrophages and axon regeneration after 7 days [10].

Inflammation following SCI has both beneficial and destructive effects on tissues. Inflammation can lead to deterioration of the extracellular matrix and extensive cell damage. In the first week after SCI, early inflammatory events create an adverse microenvironment during the treatment of various SCIs, which creates obstacles to transplant-oriented treatment. During the acute and chronic SCI stages, systemic and local inflammatory reactions can lead to neurodegenerative events, forming cavities and glial scars in the parenchyma of the spinal cord, leading to neuron and glial cell death. Eliminating the pro-inflammatory environment of an injured spinal cord has become the main therapeutic approach for reducing secondary cell death and promoting neuronal regeneration. Data from recent studies have shown that inflammation is beneficial for functional recovery and neuronal regeneration $[11,12]$. Inflammation in SCI plays an important role in clearing deteriorated and injured tissues, mediated by stimulated macrophages [13].

\section{Influence of macrophage polarization on the inflammatory microenvironment during $\mathrm{SCl}$}

After SCI, macrophages play important roles in mediating the inflammatory response during different periods.

The number of macrophages increased significantly at 3 and 7 days post-SCI injury, while macrophage-mediated inflammation peaked approximately seven days after injury [14]. Macrophages activated at the injury site can release inflammatory factors, chemokines, and mediators, and downregulate the expression of neurotrophic factors. Macrophages located in the lumbar spinal cord enhance the expression of various molecules, including $\mathrm{C}-\mathrm{C}$ motif chemokine receptor 2 (CCR2), selectin $\mathrm{L}$, and matrix metalloproteinase-9. The accumulation of these substances slows the recovery of patients' functions [15]. Macrophages can produce growth factors that promote angiogenesis, stimulate fibroblast proliferation, and regulate connective tissue synthesis, which are the key elements of tissue repair. In addition to their roles as effectors of phagocytes and tissue repair, macrophages help trigger adaptive immunity [16]. However, after SCI, the role of macrophage activation remains controversial [17]. After SCI, the integrity of the damaged tissue and the recovery of nerve function can be improved by inhibiting or depleting macrophages [18]. However, injecting pre-activated monocytes into the spinal cord can promote axon growth and accelerate the improvement of motor function [19]. Thus, macrophage polarization plays important roles in SCI and damage repair mediated by the inflammatory response.

\section{Macrophage polarization}

The term "polarization" refers to the phenomenon whereby macrophages exhibit different functional phenotypes in different microenvironments. This mainly includes "classically activated macrophages" (M1, proinflammatory) and "alternatively activated macrophages" (M2, anti-inflammatory). M1 and M2 macrophages can be produced following stimulation by different factors. They express different molecular markers and produce 
their own characteristic secretory factors, as shown in Table 1.

Within hours after SCI, macrophages (as first-response cells) become polarized into M1 macrophages after stimulation by helper $\mathrm{T}$ lymphocyte factors, such as interferon- $\gamma$ (IFN- $\gamma$ ), lipopolysaccharide, and tumor necrosis factor (TNF)- $\alpha$. The number of M1 macrophages peaks within one day after SCI. As a key signaltransduction factor, nuclear factor kappa B (NF- $\mathrm{kB}$ ) plays an important role in $\mathrm{M} 1$ macrophage polarization. When $\mathrm{NF}-\mathrm{kB}$ binds to the adenosine loop effector element binding protein (a regulatory transcription factor), it can stimulate gene transcription and cooperatively promote inflammatory gene transcription [20]. M1-type macrophages are regarded as harmful components during SCI. The induction of neuronal necrosis through various pathways and chondroitin sulfate proteoglycan expression inhibit neuron growth; pro-inflammatory M1-like macrophages produce pro-inflammatory cytokines and cytotoxic mediators induced by inflammatory factors, which simultaneously enhance their phagocytic and antigen-presentation abilities, and promote tissue destruction and microorganism killing, which in turn promotes the transformation of more macrophages to the M1 phenotype [21]. Activated M1-like macrophages express high levels of inducible nitric oxide synthase (iNOS), major histocompatibility complex II (MHC II), CD80, CD86, and CD16/CD32, and present antigens to $T$ cells to activate and regulate innate and acquired immune responses [22]. The characteristic cytokines of M1 macrophages are interleukin (IL)-6, IL-12, and IL-23 [23]. M1 macrophages produce high levels of oxidative metabolites (such as nitric oxide and superoxide) and pro-inflammatory cytokines, which are essential for host defenses, but can also cause collateral damage to healthy cells and tissues [24]. M1 macrophages also produce other rejectionrelated factors, which induce axonal retraction after SCI [25]. Other evidence has shown that M1 macrophage polarization, triggered by IFN- $\gamma$ and TNF- $\alpha$, can decrease the phagocytic ability [26], which is very important for tissue repair after SCI.

M2-type macrophages are generated via induction with TH2 cytokines (such as IL-4, IL-10, and IL-13). The molecular markers of M2 macrophages are CD206, CD163, arginase 1 (ARG1), and found in inflammatory zone 1 (Fizz1) [27]. The characteristic cytokines are IL-10 and IL-4 [28]. During SCI, these macrophages help the body resist inflammation and promote tissue regeneration. They express IL-10 and transforming growth factor (TGF)- $\beta$ and exhibit upregulated Arg1 expression. At a later stage, M2 macrophages can phagocytize scar tissue and myelin sheaths, which are harmful to nerve growth [29]. M2-like macrophages can inhibit the production of pro-inflammatory cytokines and affect macrophages by increasing MHC II expression. Transplanting M2 phenotypic macrophages or manipulating endogenous macrophages to acquire the M2 phenotype reduced spinal cord pathological injury, promoted regeneration, and improved functional recovery after SCI in rats [30]. Compared with wild-type mice, SCI mice lacking IL-10 displayed poor functional recovery [31]. Therefore, M2 macrophages have important research value for SCI treatment. However, excessive M2-like activation can promote the release of several growth factors, which in turn can promote the formation of fibrotic scars [32] and, thus, affect SCI recovery.

Given that most inflammatory events in the damaged central nervous system (CNS) occur after macrophage activation and migration, they may directly affect SCI repair, downstream inflammatory processes, secondary degeneration, and endogenous mechanisms [17]. The roles of polarized macrophages in the SCI microenvironment can be divided into advantageous and disadvantageous roles.

\section{The roles of polarized macrophages in the $\mathrm{SCI}$ microenvironment \\ Advantageous roles of polarized macrophages}

Polarized macrophages can help remove harmful substances from injured sites in the spinal cord, assist in injury repair, and play active roles in nerve recovery.

Initially, M1 macrophages infiltrate sites of injury to repair wounds and remove bacteria, foreign bodies, and dead cells. IL-12 and IL-23 secretion by M1 macrophages can directly differentiate and expand anti-inflammatory TH1 and TH17 cells, which promotes processes related to mounting an inflammatory response and clearing invasive microorganisms [33]. Activated M1-like macrophages express high levels of MHC II and present antigens to $\mathrm{T}$ cells, which activates and regulates innate

Table 1 Differences between M1 and M2 macrophages

\begin{tabular}{|c|c|c|c|}
\hline Types & Inducing factor & Molecular marker & Characteristic cytokine \\
\hline M1 macrophages & $\begin{array}{l}\text { TH1 cytokines (such as IFN- } \text {, lipopolysaccha- } \\
\text { ride, TNF-a) }\end{array}$ & $\begin{array}{l}\text { iNOS, CD16/32, CD80, CD } 86 \text {, } \\
\text { MHC } \|\end{array}$ & IL-1 $\beta,\|L-12\| L-6,, I L-23$, TNF-a, IFN- $\gamma$ \\
\hline M2 macrophages & TH2 cytokines (such as IL-4, IL-10, IL-13) & CD206, CD163, Arg1, Fizz1, YM1 & IL-4, IL-10, IL-13, TGF- $\beta$ \\
\hline
\end{tabular}


acquired immune responses [22]. The phagocytic and antigen-presentation abilities of M1-polarized macrophages are enhanced, which promotes the clearance of necrotic cells [34].

TGF- $\beta$ and IL-10 are important anti-inflammatory factors expressed by M2 macrophages. These factors can promote the regeneration and neuroprotection of injured spinal cord tissue, as well as the renewal of damaged progenitor cells [13]. Accordingly, M2-like macrophages produce IL-10, the IL-1 receptor antagonist (IL-1Ra), and various chemokines, which induce tissue remodeling and promote the regeneration and growth of adult sensory nerve axons [24]. Some data have shown that transplanting M2 phenotypic macrophages may cause endogenous macrophages to acquire the M2 phenotype, mediate tissue remodeling, reduce pathological SCI, promote regeneration, and assist in functional recovery after $\mathrm{SCI}$ in rats. Therefore, M2 macrophages play active roles in repair and regeneration after SCI $[30,35]$.

\section{Disadvantageous roles of polarized macrophages}

Following SCI, necrotic cells release superoxide dismutase, which can induce macrophages to produce cytokines, such as IL-23, which accelerate nerve cell death [36]. In addition, M1 macrophages produce numerous inflammatory substances, such as reactive oxygen species (ROS) and reactive nitrogen species (RNS) through oxidative reactions, which inhibit cellular oxidative defenses and lead to further oxidative stress and SCI exacerbation [37].

M1 macrophages are neurotoxic [24]. They inhibit the establishment of the blood-brain barrier (BBB), aggravate lesions, promote cell death, and intensify immune responses. M1 macrophages also hinder the regeneration of the CNS [38]. Specifically, inflammatory factors (such as TNF- $\alpha$, IL- $\beta$, ROS, RNS, prostaglandin 2 , and other active substances) are secreted and released after M1 macrophage polarization, which damage neurons and glia, and even cause neuronal apoptosis [34, 39]. For example, cytotoxic ROS react with polyunsaturated fatty acids to cause lipid oxidation and degradation, which affect the fluidity and permeability of cell membranes and hinder cell metabolism and ion-channel exchange [40]. In addition, iNOS was highly expressed in lesions after SCI $[41,42]$. Previous data also showed that ROS, RNS, and inflammatory substances produced by $\mathrm{M} 1$ macrophages can cause tissue damage [33].

\section{Regulatory mechanism of macrophage polarization}

Macrophage polarization is regulated by various signaling molecules and their related pathways. At present, the main signaling pathways include the phosphatidylinositol-3-kinase (PI3K)-protein kinase B (Akt) signaling pathway, the Notch-signaling pathway, the Janus kinase (JAK)-signal transducer and activator of transcription (STAT)-signaling pathway, the TGF- $\beta$-signaling pathway, and the Toll-like receptor (TLR) 4-NF-kB-signaling pathway (Fig. 1).

\section{PI3K-Akt-signaling pathway}

Regarding the PI3K-Akt pathway, it is necessary to first understand the main regulatory factors, namely PI3K and Akt. PI3K is an intracellular phosphatidylinositol kinase that is activated by cytokine receptors and TLRs [43]. The AKT family consists of three serine-threonine kinases, namely Akt1, Akt2, and Akt3 [44]. PI3K can produce phosphatidylinositol $(3,4,5)$-triphosphate and activate protein kinase Akt, which induces macrophage polarization by regulating microRNA-155 (miR-155) expression. Specifically, Akt1 promotes the formation of the M2 phenotype, and Akt2 promotes the formation of the M1 phenotype [45]. In mechanistic terms, Akt2 enhances miR-155 expression at the transcriptional level, which leads to primary (pri)-miRNA formation, subsequent processing to pre-miRNA, followed by the production of mature miRNA. Following increased pri-miR-155 production, miR-155 expression increases, which interferes with the cAMP-response element binding protein (CREB)-CCAAT-enhancer-binding protein $\beta$ $(C / E B P \beta)$ cascade, weakens M2-specific gene upregulation, and simultaneously downregulates $C / E B P \beta . C / E B P \beta$ is a member of the C/EBP basic-region leucine zipper protein family, which can transcriptionally activate the IL-10 and Arg1 promoters. The IL-10 and Arg1 promoters induce M1 macrophage polarization [46]. In addition, increased miR-155 expression also inhibited synthesis of the cytokine signal-transduction inhibitor 1 (SOCS1) protein, which is an important regulator of M1/M2 macrophage polarization, which promoted formation of the M1 phenotype [47, 48]. In contrast, Akt1 inhibits miR155 expression, thus increasing the expression of $\mathrm{C} / \mathrm{EBP} \beta$ and genes related to the M2 phenotype (ARG1 and IL-4), in turn promoting M2 macrophage polarization [46].

\section{Notch homolog 1, translocation-associated (Notch)-signaling pathway}

Four different Notch receptors (Notch1, Notch2, Notch3, and Notch4) participate in the mammalian Notch-signaling pathway and are expressed in various tissues and organs. Notch receptors are comprised of a single transmembrane domain with functional extracellular, transmembrane, and intracellular domains. Notch ligands can be divided into Delta-like (DLL1, DLL3, and DLL4) and Jagged (JAG1 and JAG2) families [49]. These ligands bind to the same or different Notch receptors and activate them, which stimulates the 


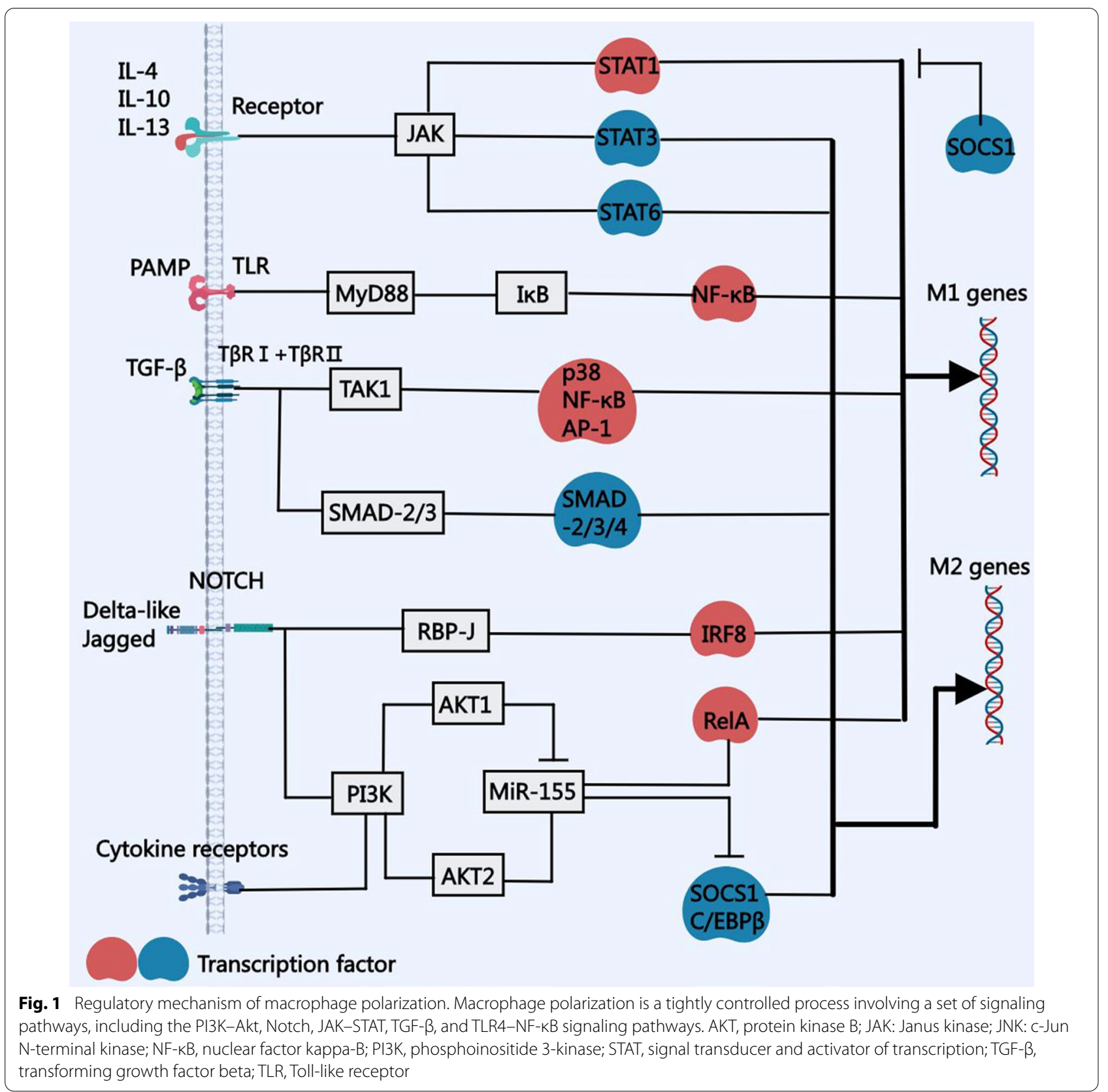

Notch-signaling pathway. The Notch-signaling pathway triggers many regulatory effects in terms of macrophage polarization, which are described below:

(1) Mechanistically, the Notch-signaling pathway mainly activates the recombination signal binding protein for immunoglobulin kappa J region (RBP-J) transcription factor and interferon regulatory factor (IRF)8, which reprograms the M1 and M2 phenotypes of macrophages through the PI3K pathway. Macrophages expressing DLL4 receptors can induce Notch proteolysis, resulting in increased IL-12 gene activity, which in turn induces macrophages to acquire the M1 phenotype [50].

(2) RBP-J is an important transcription factor in the Notch-signaling pathway, and Notch1 activation can promote RBP-J production. RBP-J increases IRF8 expression in macrophages. Importantly, IRF8 not only participates in the Notch-signaling pathway, but also activates the TLR-4-signaling pathway to produce pro-inflammatory M1 cytokines [51]. Compared with non-reprogrammed macrophages, with an increased IRF8 content, the combined effects of ligands and TLR-4 on reprogrammed 
macrophages can lead to more significant inflammatory reactions, which is a pro-inflammatory feature of reprogrammed macrophages [52].

(3) The Notch1-signaling pathway can also promote formation of the pathogenic M2 phenotype [53]. In this case, the Notch1-signaling pathway translates signals through the PI3K pathway, activates genes related to the M2 phenotype, and reprograms macrophages to acquire the M2 phenotype [54].

(4) The STAT gene is also important for the Notchsignaling pathway. The STAT gene is encoded on chromosome 17 and is a kind of proto-oncogene. SOCS3 is an M2 transcription factor that inhibits expression of the STAT3 gene. STAT expression can lead to increased Notch-RBP-J pathway-dependent reprogramming of macrophages to the M1 phenotype. STAT function represents the mechanism between increased proinflammatory cytokine production and decreased antiinflammatory cytokine production [53].

\section{JAK-STAT-signaling pathway}

JAKs are non-receptor tyrosine protein kinases that are activated by various cytokines. Downstream target genes are activated by STATs, and the corresponding expressed proteins play regulatory roles. The JAK-STAT pathway is mainly composed of three parts: tyrosine kinase-related receptors, JAKs, and STATs. Four types of JAKs have been discovered, namely JAK1-3 and tyrosine kinase 2 (TYK2). In addition, six types of STATs are known (STAT1-6). The SH2 structural region of STAT proteins is identical to the corresponding core sequence in JAK proteins, which is responsible for recognizing specific JAKs. The basic signal-transduction pathway involves cytokine-receptor binding on the cell surface, receptor dimerization, and JAK polymerization and phosphorylation. Activated JAKs can bind the SH2 domains of STATs, become activated after STAT phosphorylation, and finally enter the nucleus in the form of a homodimer or a heterodimer to promote the transcription of target genes [55]. The JAK-STAT pathway regulates many effects in terms of macrophage polarization, as described below:

(1) IFN- $\gamma$ induces STAT1 activation through the JAKSTAT pathway. STAT1 then acts as a homodimer to bind the cis element (known as the IFN- $\gamma$-activation site) in the promoters of genes related to M1 polarization, resulting in increased production of pro-inflammatory cytokines and acquisition of the M1 phenotype [56]. The IFN- $\gamma$-JAK-STAT1 pathway is controlled by IRF4 and IRF5 [57]. They have opposite effects in terms of the IFN- $\gamma$-JAK-STAT1 pathway and macrophage phenotype. IRF 5 can be activated by pro-inflammatory factors, whereas IRF4 can be activated by anti-inflammatory factors [58]. IRF5 promotes the production of IL-12, an M1 cytokine dependent on the IFN- $\gamma-J A K-S T A T 1$ pathway, whereas IRF4 inhibits the effects of IRF5 [58].

(2) IL-4, IL-13, and IL-10 polarize macrophages to the M2 phenotype through the JAK-STAT-signaling pathway. IL-4 binds to its receptor to activate JAK. Then, two transcription factors of genes related to the M2 phenotype (STAT3 and STAT6) are phosphorylated and activated [59]. In addition, IL-4 can also induce c-Myc gene expression, which in turn increases the expression of M2-phenotype genes, such as scavenger receptor class $\mathrm{B} 1$ and mannose receptor, $\mathrm{C}$ type 1 , as well as the activity of STAT6 and peroxisome proliferator-activated receptor (PPAR) $-\gamma[60]$.

(3) IL-13 binds to receptors to activate the JAK1, JAK2, and TYK2 kinases, and then activates STAT1, STAT3, and STAT6. STAT3 and STAT6 activate the expression of M2-phenotype genes, such as the mannose receptor, Fizz1, chitinase-like protein 3 (YM1), and anti-inflammatory cytokines, whereas STAT1 activates pro-inflammatory cytokines. The anti-inflammatory response of macrophages induced by IL-13 may reflect the fact that STAT3 and STAT6 are more extensively activated than STAT1 [61].

(4) IL-10 binds to receptors to activate JAK1 and STAT3, resulting in activation of M2-phenotype genes, such as TGF- $\beta$ and IL-10 [61].

(5) The SOCS1 and SOCS3 proteins are two important regulators of the JAK-STAT-signaling pathway during M1/M2 macrophage polarization [47]. IL-4 activates SOCS1 synthesis and blocks STAT1 production, thus preventing formation of the M1 phenotype. IFN- $\gamma$ and TLR4 ligands activate SOCS3 synthesis, which blocks STAT3, thus preventing the formation of the M2 phenotype [62]. In addition, SOCS1 activated the M2 phenotype-related transcription factor STAT6, while SOCS3 activated M1 phenotype-related transcription factor STAT1. These interactions between SOCS and STAT further explain the relationship between the increase of pro-inflammatory cytokines and the decrease of antiinflammatory cytokines produced by macrophages during polarization to the M1 phenotype [44].

\section{TGF- $\beta$-signaling pathway}

The TGF- $\beta$ protein family includes TGF- $\beta 1$, TGF- $\beta 2$, TGF- $\beta 3$, activin, and several growth factors. Macrophages mainly produce TGF- $\beta 1$. The TGF- $\beta$ receptor is composed of two type-I transmembrane subunits (T $\beta R I)$ and two type-II subunits (T $\beta R I I)$ with a cytoplasmic kinase domain. After TGF- $\beta$ binds with its receptor, T $\beta$ RII is auto-phosphorylated and then phosphorylates T $\beta R I$. Next, the T $\beta R I$ cytoplasmic kinase domain binds to and phosphorylates (activates) SMAD2 and SMAD3. Activated SMAD2 and SMAD3 bind to SMAD4, and the 
resulting ternary complex translocates into the nucleus, which upregulates the activities of the M2 phenotyperelated genes ARG1 and MGL2, and reprograms macrophages to acquire the M2 phenotype [63].

In addition, TGF- $\beta$-dependent reprogramming of the M2 phenotype is related to SMAD7. SMAD7 can bind to T $\beta R I$, which prevents SMAD2 and SMAD3 phosphorylation, and can direct both SMADs to the proteasome for degradation. The proinflammatory factors IFN $-\gamma$ and TNF- $\alpha$ can upregulate SMAD7 expression, thus inhibiting the TGF- $\beta-$ SMAD pathway and reducing the production of anti-inflammatory factors. This mechanism can help explain the relationship between increased production of pro-inflammatory factors and decreased production of anti-inflammatory factors [64].

In addition to the SMAD-dependent TGF- $\beta$-signaling pathway, TGF- $\beta$ can also activate the SMAD-independent TGF- $\beta$-signaling pathway. In the SMAD-independent pathway, the TGF- $\beta$-activated kinase protein 1 (TAK1) transduces signals from TGF- $\beta$ to several downstream signal cascades, including the transcription factors JNK, p38, and NF-kB [44]. These SMAD-independent pathways can reprogram macrophages to acquire the M1 phenotype. When the SMAD-dependent pathway is blocked, the effect of the SMAD-independent pathway is more pronounced [65].

\section{TLR4-NF-KB-signaling pathway}

TLRs belong to the pattern-recognition receptor transmembrane family, and six TLRs (TLR1, TLR2, TLR4, TLR5, TLR6, and TLR10) have been identified on the surface of macrophages. TLRs can identify specific pathogen-associated molecular patterns (PAMPs) on microbial molecules. PAMP binding to TLRs triggers signal cascades, which induce macrophage reprogramming into the M1 phenotype [66]. When PAMPs bind to TLRs, they dimerize, which activates myeloid differentiation primary response protein 88 (MyD88) [66]. MyD88 combines with members of the IL-1R related kinase (IRAK) family to form the Myddosome complex [67].

IRAK is phosphorylated in the Myddosome complex, phosphorylated IRAK attracts tumor necrosis factor receptor related factor-6 (TRAF6) to the cell membrane, and TRAF6 can attract the TAK1 complex [68].

TRAF6 attracts the TAK1 complex, which causes autophosphorylation and activation of TAK1 kinase, which in turn activates the IKB kinase (IKK) complex [69]. IкB functions as an inhibitory subunit related to the NF- $\mathrm{kB}$ transcription factor in the cytoplasm of macrophages. IKB can be phosphorylated by IKK, which leads to its degradation in proteasomes. Free NF- $\mathrm{KB}$ is transported to the nucleus, where it activates genes related to inflammation, immune responses, and cell growth [69].
There are five proteins in the NF- $\mathrm{kB}$ family in mammals, namely RelA (p65), RelB, c-Rel, NF-kB1 (p50), and NF- $k B 2$ (p52). Proteins belonging to the p65, RelB, and c-Rel families can induce macrophage reprogramming into the M1 phenotype, whereas proteins belonging to the p50 and p52 Relish families have no such effect [70]. Pro-inflammatory cytokines produced by the NF-kBdependent pathway can repeatedly activate the NF- $\mathrm{kB}-$ dependent pathway and form a positive-feedback loop, such that macrophages rapidly acquire the M1 phenotype. NF- $\kappa B$ can also activate the IкB gene, limit excessive nuclear translocation of NF- $\mathrm{kB}$, and act as a negativefeedback mechanism to prevent excessive inflammation [71].

In summary, macrophage polarization is highly regulated by various molecules and signaling pathways. Defining the key pathways and regulatory factors that regulate macrophage polarization is key for promoting the polarization of pro-inflammatory M1 macrophages into antiinflammatory M2 macrophages and, thus, improving the unfavorable inflammatory microenvironment after SCI and promoting SCI repair. MSCs, as a source of adult stem cells, are easy to obtain. The regulation and mechanism of MSCs on SCI repair and macrophage polarization have been expounded upon, and such insights have important guiding significance for applying MSCs to autologous stem cell therapy after SCI.

\section{MSCs alleviate $\mathrm{SCl}$ by inducing macrophage/microglial cell polarization}

MSC transplantation-an effective way to repair SCI

SCI can result in severe disabilities associated with motor, sensory, and autonomic dysfunctions. Stem cell transplantation is considered a potential therapy for stimulating neural plasticity and nerve regeneration after SCI [72]. MSC transplantation is a promising approach for treating SCI. It can be widely used in clinical practice because, compared with other stem cells, such as embryonic stem cells and induced pluripotent stem cells, MSCs are more easily obtained, and few ethical and safety concerns are associated with autologous transplantation [73]. MSC-derived neural network tissue transplanted in this way can be used as a "neuronal relay" of structure and function to restore the motor functions of paralyzed limbs of organisms with complete SCI [74]. In addition, transplanted MSCs can not only provide tissue replacement, but can also release the nutrients and matrix components needed for tissue regeneration. Transplanted MSCs also exert anti-inflammatory effects by downregulating pro-inflammatory factors [75]. Evaluating histopathological changes, proinflammatory cytokine levels, and locomotor functions, and verifying the effects of exogenous bone marrow stromal cell (BMSC) lines and 
BMSCs on animals with SCI have revealed that intravenous BMSCs have good therapeutic effects [76]. In addition, the current main functions of cell therapy include restoration of sphincter dysfunction and relief from neuropathic pain, which are very safe and effective for use in treating patients with SCI [77].

At present, MSC-based SCI treatment mainly includes intravenous injection of MSCs, intravenous injection of BMSC-derived exosomes, and transplantation of BMSCs + scaffold materials.

Intravenous infusion of BMSCs promoted functional recovery after contusive $\mathrm{SCI}$ in non-immunosuppressed animals following local treatment [78], which can provide neuroprotection, stabilize the blood-spinal cord barrier (BSCB), regenerate the myelin sheath, and germinate axons. Compared with other cell types, BMSCs are ideal transplantable cells and mainly regulate the SCI cascade through a paracrine mechanism. After BMSCs were infused, the myelin sheath regenerated widely around the focus center, and the sprouting of corticospinal tract and serotonergic fibers increased. In addition, systemic infusion of BMSCs can lead to functional improvement related to structural changes in chronically injured spinal cords, including BSCB stabilization, axonal germination and regeneration, and remyelination [79].

In addition, transcranial magnetic stimulation (alone or together with human umbilical cord blood MSC transplantation) alleviated neural stem cell apoptosis and motor dysfunction induced by SCI [80]. Ischemic SCI (ISCI) is a devastating complication of aortic surgery with few preventive strategies. Intravenous BMSC infusion has been shown to provide functional improvement for patients with ISCI. Potential therapeutic mechanisms include the neuroprotection of white and gray matter and damaged spinal cord, reduction of axon loss or degeneration, and reduction of BSCB damage in the damaged spinal cord. Therefore, BMSC therapy may have therapeutic value in ISCI [81].

SCI leads to a strong inflammatory reaction, which greatly affects the functions of stem cells [82]. Whether scaffolds can be used as adjuvant therapies for MSC transplantation in SCI has long been controversial. Therefore, a meta-analysis of preclinical evidence was conducted to evaluate the effectiveness of stent + MSC transplantation in improving SCI motor dysfunction, compared with stent or MSC treatment alone. The results showed that, in the acute-injury stage, after SCI, stent + MSC treatment was more effective in improving motor functions than using a stent and MSCs alone [83].

\section{The mechanism whereby MSCs regulate macrophage polarization in the context of $\mathrm{SCl}$}

The results of several studies have shown that MSC transplantation after SCI can promote the polarization of macrophages/microglia from the M1 phenotype to the M2 phenotype, improve the inflammatory environment, and promote recovery after SCI $[84,85]$. It was reported that TNF- $\alpha$ produced by the spleen plays an important role in activating transplanted MSCs, suggesting that the spleen is involved in MSC-mediated effects [86]. Furthermore, compared with allogeneic MSCs, syngeneic MSCs have superior therapeutic potential [87]. Combined treatment with biomaterial and MSCs may provide a promising therapeutic treatment for SCI. For example, Zhan et al. showed that MSCs loaded on a nerve-guided collagen scaffold could better promote M2 macrophage polarization [88]. MSCs combined with a three-dimensional biomimetic hydrogel could deliver cytokines to the SCI site, thereby increasing the M2 macrophage population and promoting a pro-regenerative environment [89].

In terms of adjustment methods, MSCs can induce macrophage polarization by secreting soluble factors or exosomes in the context of SCI (Fig. 2).

\section{Secretion of soluble factors}

MSCs can regulate macrophage polarization by secreting soluble proteins, including ILs and chemokines, which ultimately relieve SCI.

Multiple findings have shown that ILs play important roles in macrophage polarization. IL-4 and IL-13 are classic inducers of the M2 macrophage phenotype [90, 91]. IL-4 induces c-Jun N-terminal kinase (JNK) in macrophages, leading to subsequent downstream transcription of c-Myc in conjunction with IL-4R $\alpha$, as well as increased expression of the M2 markers, Arg1 and Mrc1 [92, 93]. In addition, IL-4 upregulates the expression of M2 macrophage genes (such as Arg-1 and YM1) by activating the IL4-R $\alpha-J A K-S T A T 6$ and PI3K pathways, which facilitate M2 macrophage polarization. PPAR $\gamma$ and PPAR $\delta$ also participate in the process as downstream signals of STAT6 $[94,95]$. IL-13 acts on a complex receptor composed of IL-4R and IL-13R $\alpha$, and upregulates M2 macrophage markers by activating STAT6 to promote macrophage polarization [95]. Researchers transplanted MSCs genetically engineered to secrete IL-13 into mice with SCI and observed a significant increase in M2 macrophages in the transplanted areas. It is worth noting that the majority of these are peripherally derived macrophages [96]. Furthermore, using an anti-IL-7R $\alpha$ monoclonal antibody to block IL-7 signal transduction after SCI can promote M2 macrophage induction [97]. Another study provided evidence that transplanted human umbilical cord MSCs can promote M2 macrophage polarization by decreasing IL-7 expression, thereby alleviating SCI [98].

Research conducted by Tomomi et al. showed that implanting human MSCs into mice with SCI induced M2 macrophages through a mechanism related to IL-4 


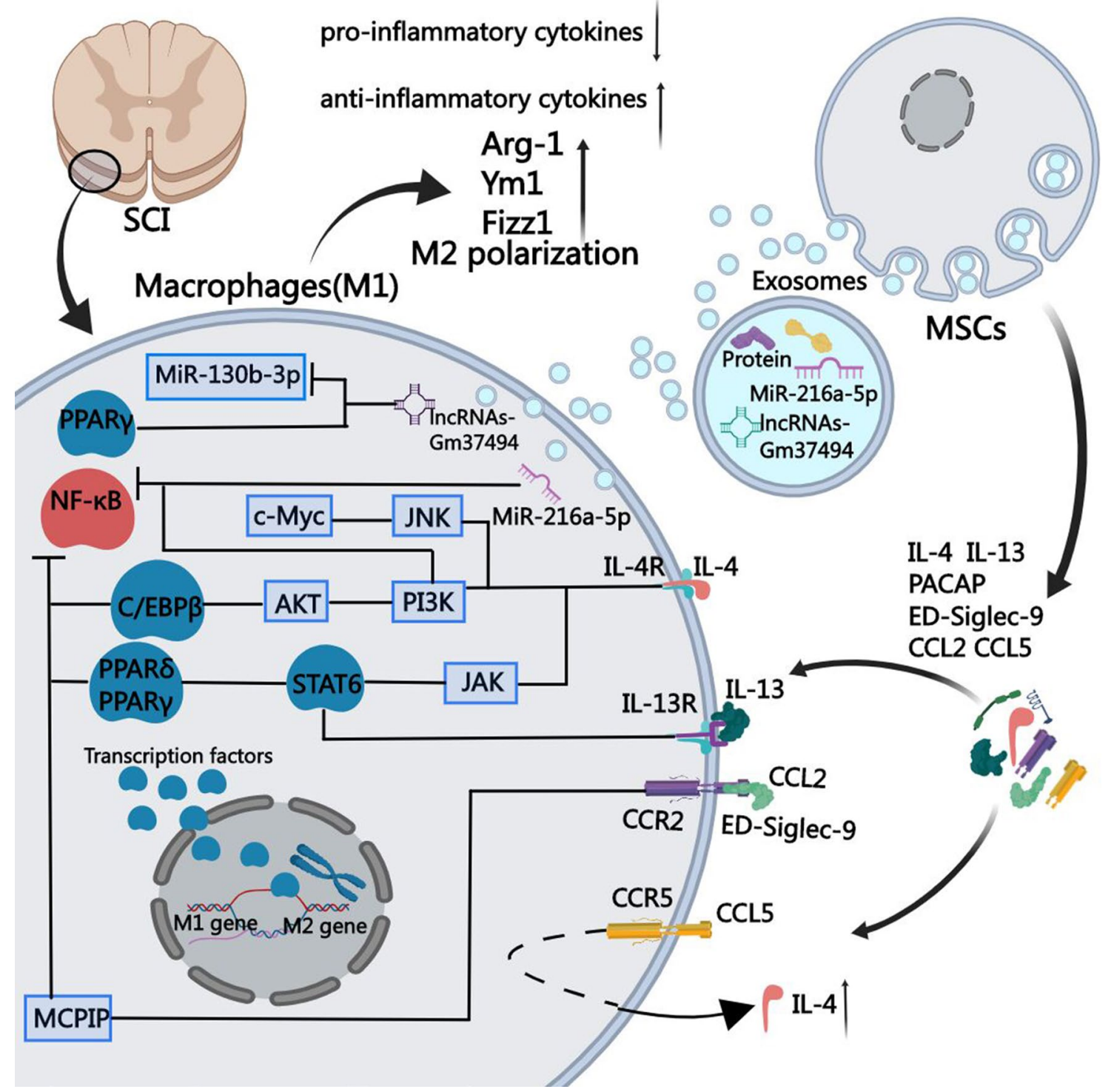

Fig. 2 Mechanism by which MSCs regulate macrophage polarization in the context of SCI. MSCs can promote the polarization of macrophages at the site of SCI by secreting soluble proteins including IL-4, IL-13, PACAP, CCL2, CCL5, and ED-Siglec-9. IL-4 can activate the JNK, JAK/STAT6, and PI3K signal pathways through IL-4R, and ultimately promote the expression of M2 related genes. In turn, IL-13 activates IL-10R, thereby participating in the process by acting on STAT6. In addition, CCL5 can induce high levels of IL-4 by acting on CCL5R, thereby promoting M2 polarization of macrophages. CCL2 can induce the expression of MCPIP through CCR2, thereby activating C/EBP $\beta$, PPARY, and inhibiting NF-kB to exert anti-inflammatory effects. ED-Siglec-9 also works synergistically with CCR2. Regarding PACAP, its mechanism of action is not yet clear. Furthermore, MSCs-exo can also play a similar role, by carrying MiR-216a-5p and IncRNAs Gm37494, which will inhibit TLR4/NF-kB and activate PI3K/AKT signaling pathway, and downregulate the expression of MiR-130b-3p and upregulate the expression of PPARy, respectively, hence, inhibiting M1 and enhancing M2 marker expression. Lastly, MSCs can improve the inflammatory environment of the $\mathrm{SCl}$ site and relieve its manifestations. AKT, protein kinase B; CCL, chemokine (C-C motif) ligand; CCR, chemokine (C-C motif) receptor; C/EBP $\beta$, CCAAT/enhancer-binding protein beta; ED-Siglec-9, ectodomain of sialic acid-binding Ig-like lectin-9; IL, interleukin; JAK, Janus kinase; JNK, c-Jun N-terminal kinase; IncRNAs, long non-coding RNAs; MCPIP, monocyte chemoattractant protein-1-induced protein.; MSCs, mesenchymal stem cells; NF-KB, nuclear factor kappa-B; PACAP, pituitary adenylate cyclase-activating polypeptide; PI3K, phosphoinositide 3-kinase; PPAR $\beta$, peroxisome-proliferator-activated receptor $\beta$; SCI, spinal cord injury; TLR, Toll-like receptor

upregulation. The neuropeptide, pituitary adenylate cyclase-activating polypeptide, induced by the microenvironment also participates in crosstalk between MSCs and macrophages and enhances macrophage polarization, although the specific mechanism involved remains unclear [99]. 
Chemokines promote the migration of immune cells and participate in inflammatory reactions, which can be used to research communications between MSCs and macrophages [100]. Chemokine (C-C motif) ligand 2 (CCL2), also known as monocyte chemotactic/chemoattractant protein 1 (MCP1), is an inflammatory chemokine produced by monocytes [101]. CCL2 functions by binding to CCR2 and initiating intracellular signal transduction [102]. Elena et al. showed that CCL2 and CCR2 are differentially expressed in macrophages. Compared with M1 macrophages, M2 macrophages express higher levels of CCL2; however, CCR2 surface expression is only observed with M1 macrophages. For this reason, only M1 macrophages can respond to CCL2 stimulation [103]. Previous findings have shown that CCL2 can not only recruit peripheral pro-inflammatory M1 macrophages, but can also promote M2 macrophage polarization $[104,105]$. The specific mechanism involves the targeted binding of CCL2 and CCR2, and a series of downstream changes, including increased activation of p38, ERK1/2, MSK1/2, HSP27, JNK, and STAT5a/b [103], increased activation of the p42 and 44 mitogenactivated protein kinases [103, 106], and upregulation of MCP-1-induced protein (MCPIP), which inhibits NF- $\mathrm{BB}$ activation and induces C/EBP $\beta$ and PPAR $[107,108]$. Researchers have used biomimetic hydrogel scaffolds encapsulated with MSCs to treat SCI in mice. The results showed that CCL2 secreted by MSCs can be effectively transferred to the diseased spinal cord, leading to downregulated expression of M1 markers (TNF- $\alpha$ and IL-1 $\beta$ ) and upregulated expression of M2 markers (YM1 [also known as Chil3], is a member of the chitinase-like protein family) and Arg-1, which promoted peripheral macrophage M2 polarization [109]. In another study, MCP-1 and a previously unrecognized inducer of M2 macrophages were analyzed in the medium of MSCs, which revealed secretion of the ectodomain of sialic acid-binding Ig-like lectin-9 and synergistic induction of M2-like macrophages through CCR2 [105]. In addition, research conducted using a SCI mouse model showed that transplanted MSCs can cause neuronal cells to secrete CCL2 and induce CCR2 expression in granulocytes. Through targeted CCL2-CCR2 binding, MSCs can increase macrophage expression of the zinc finger $\mathrm{CCCH}$-type containing 12A protein (encoded by MCPIP and involved in M2 polarization), which can inhibit NF- $\mathrm{KB}$ signaling and ultimately induce $\mathrm{M} 2$ polarization and promote recovery from $\mathrm{SCI}$ in rats [110]. Chemokine ( $\mathrm{C}-\mathrm{C}$ motif) ligand 5 (CCL5), also known as RANTES, is one of the members of the mammalian chemokine system [111]. CCL5 is usually studied as a marker of M1 macrophage polarization because it acts on multiple receptors, both typical (CCR1, CCR3, CCR5, and CCR4) and atypical (non-signaling receptors like ACKR1, ACKR2, and CCRL2) [112, 113]. The above research also demonstrated that transplanted MSCs caused neuronal cells to secrete CCL5, which bound CCR 5 on the surface of macrophages. IL- 4 is the main regulator of macrophage polarization [91]. CCL5CCR5 binding can promote M2 polarization by inducing high levels of IL-4 and upregulating the expression of Arg-1 and YM1, which are markers of M2 macrophages. In addition, CCL5-CCR5 binding can promote recovery from SCI [110].

\section{Exosomes}

Exosomes are membrane-like lipid vesicles $(30-100 \mathrm{~nm}$ in size), which contain mRNAs, miRNAs, long noncoding RNAs (lncRNAs), and proteins, and play important roles in information transfer between cells $[114,115]$. Exosomes can bind to target cells and release their contents through specific cell-surface ligands, thereby regulating specific biological functions, such as immune responses and angiogenesis [116, 117]. Data from previous studies have shown that MSCs can alleviate myocardial ischemia-reperfusion injury, myocardial infarction, sepsis, stroke, and neurological injury by secreting exosomes to induce macrophage polarization [118-121].

Regarding SCI, previous research showed that intravenous MSC-derived exosomes (MSC-exos) can specifically target M2-type macrophages at the SCI site [78]. Zhao et al. showed that MSC-exos mainly gather at the SCI site and bind to microglia (to inhibit complement release) and SCI-activated NF- $\mathrm{KB}$ (to inhibit inflammatory damage) [122]. These findings suggest that MSC-exos may mediate some of the functional roles of MSCs in SCI.

MSC-exos also alleviate SCI by participating in macrophage polarization. In an SCI model, MSC-exos promoted functional recovery by inducing the mRNA expression of M2 macrophage markers, including CD206, IL-10, and Arg-1, and facilitated bone marrowderived macrophage polarization from an $\mathrm{M} 1$ to an $\mathrm{M} 2$ phenotype. In addition, MSC-exos also decreased the levels of pro-inflammatory cytokines (TNF- $\alpha$, IL-6, granulocyte colony-stimulating factor, IFN- $\gamma, \mathrm{MCP}-1$, and macrophage inflammatory protein- $1 \alpha$ ), while increasing the levels of the anti-inflammatory cytokines, IL- 4 and IL-10 [123].

Oxygen concentration plays an important role in the processes of MSC proliferation, differentiation, and selfrenewal [124]. However, the oxygen concentration under in vitro culture conditions (21\%) is much higher than that in the body under physiological conditions $(\leq 2-8 \%)$. Hypoxic pretreatment of MSCs (to simulate the hypoxic environment in the body) can significantly improve their biological functions and activities, thereby improving the efficacy of treatment in various disease models [125, 
126]. Correspondingly, exosomes secreted by MSCs with hypoxic pretreatment showed better repair effects than the exosomes secreted by normal MSCs, suggesting that hypoxia preconditioning is a promising and effective approach for improving the efficacy of MSC-exos [127]. Liu et al. showed that hypoxia-preconditioned MSCexos were rich in miR-216a-5p, which was transferred to microglia and targeted expression of the TLR4 gene, thereby inhibiting the TLR4-NF- $\mathrm{kB}$ pathway and activating the PI3K-AKT pathway, eventually promoting polarization of microglia to alleviate SCI [128]. Shao et al. reported that exosomes secreted by hypoxia-pretreated human amnion-derived MSCs (HExos) showed inhibited expression of inflammatory factors and increased polarization of microglia from M1 to M2, which promoted functional recovery after SCI. Importantly, high-throughput sequencing analysis revealed high expression of the lncRNA, Gm37494, in HExos, which can downregulate miR-130b-3p expression and upregulate PPAR $\gamma$ expression. The former can promote the expression of inflammatory factors, and the latter can inhibit the NF- $k B$ light-chain enhancer (related to STAT activity) to inhibit NF- $\mathrm{kB}$ and the expression of M1 markers $[129,130]$.

\section{Summary and discussion}

A series of pathophysiological changes occur after SCI. The primary injury is related to the destruction of axons and neurons. The secondary injury is caused by nerve inflammation, which directly or indirectly controls the sequelae of SCI and can lead to morphological edema, cavitation, and reactive glial hyperplasia. SCI treatment is challenging because it can lead to many irreversible pathological reactions. Given that the immune response in SCI is a "double-edged sword," beneficial aspects should be promoted during treatment, rather than completely inhibiting inflammation. The pro-inflammatory and anti-inflammatory effects of macrophages during different stages of SCI are important causes of symptoms at different periods. The direction of polarization can differ in macrophages, and many pathways and cytokines are involved in regulating polarization. The representative pathways are the PI3K-Akt-signaling pathway, the Notch-signal pathway, the JAK-STAT-signal pathway, the TGF- $\beta$-signaling pathway, and the TLR4-NF- $\mathrm{kB}-$ signaling pathway. MSCs have great potential for spinal cord repair and represent promising candidates for long-term treatment of secondary SCI, caused by neuroinflammation.

Although many studies involving SCI treatment have been based on BMSCs, some problems remain. For example, MSCs transplanted into the spinal cord have a low survival rate and may differentiate into other types of cells, such as osteoblasts, which limits the therapeutic effect of BMSCs. BMSCs play an important therapeutic role through exocrine secretions, and the direct application of exocrine secretions as a therapeutic agent is one research direction [131]. However, the lack of exocrine-production capacity and its low targeting are the main factors that limit this strategy at present. Moreover, applying conditioned medium from BMSCs represents an alternative method for MSC transplantation in SCI treatment, but this method is still under development [132]. Many drugs and treatments have proven effective in experimental studies, but their actual clinical effects are unknown. Beyond macrophages, $\mathrm{T}$ cell reduction can improve the recovery of the spinal cord structure and limb function after SCI, but the related mechanisms remain unclear [133135]. The beneficial and detrimental effects of $B$ cells before and after SCI damage to the BBB remains controversial [136]. In future research, dynamic changes in the immune system after SCI should be explained. Further exploration and experiments are needed to coordinate the interaction between different cells and improve the efficacy of nerve injury treatment. In addition to exploring the strategy of directly applying MSCs, treatment with BMSCs should also be studied in terms of specific functional exocrine secretions or conditioned MSC medium, in combination with other clinical strategies to lay the foundation for improved practical applications.

\section{Acknowledgements \\ Not applicable.}

\section{Authors' contributions}

$N A, J Y, H W, S S$, and HW wrote the original draft of the manuscript. LL and ML contributed to the conceptualization and provided the funding. All authors read and approved the final manuscript.

\section{Funding}

This work was supported by the Outstanding Youth Program for Science and Technology Development of Jilin Province (Grant No. 20190103094JH), the National Natural Science Foundation of China (Grant No. 31201052), and the Health Technology Innovation Project of Jilin Province (Grant No.2018j064).

\section{Availability of data and materials}

The datasets used and/or analyzed during the current study are available from the corresponding author on reasonable request.

Ethics approval and consent to participate

Not applicable.

\section{Consent for publication}

Not applicable.

\section{Competing interests}

The authors declare that they have no competing interests.

\section{Author details}

${ }^{1}$ The Key Laboratory of Pathobiology, Ministry of Education, Jilin University, 126 Xinmin Street, Changchun 130021, Jilin, People's Republic of China. ${ }^{2}$ The Second Hospital of Jilin University, Changchun 130021, Jilin, China. ${ }^{3}$ The First Hospital of Jilin University, Changchun 130021, Jilin, China. 
Received: 7 November 2020 Accepted: 11 February 2021

Published online: 23 February 2021

\section{References}

1. Pukos N, et al. Myelin status and oligodendrocyte lineage cells over time after spinal cord injury: what do we know and what still needs to be unwrapped? Glia. 2019:67(11):2178-202.

2. Romanelli $\mathrm{P}$, et al. Extracellular vesicles can deliver anti-inflammatory and anti-scarring activities of mesenchymal stromal cells after spinal cord injury. Front Neurol. 2019;10:1225.

3. Qian D, et al. Blocking Notch signal pathway suppresses the activation of neurotoxic $\mathrm{A} 1$ astrocytes after spinal cord injury. Cell Cycle. 2019;18(21):3010-29.

4. Sabirzhanov B, et al. Inhibition of microRNA-711 limits angiopoietin-1 and Akt changes, tissue damage, and motor dysfunction after contusive spinal cord injury in mice. Cell Death Dis. 2019;10(11):839.

5. Han $\mathrm{Q}$, et al. Descending motor circuitry required for NT-3 mediated locomotor recovery after spinal cord injury in mice. Nat Commun. 2019;10(1):5815.

6. Zhao $L$, et al. Dexmedetomidine attenuates neuronal injury after spinal cord ischaemia-reperfusion injury by targeting the CNPY2-endoplasmic reticulum stress signalling. J Cell Mol Med. 2019;23(12):8173-83.

7. Carpenter RS, et al. Human immune cells infiltrate the spinal cord and impair recovery after spinal cord injury in humanized mice. Sci Rep. 2019;9(1):19105

8. Zhou X, et al. Microglia and macrophages promote corralling, wound compaction and recovery after spinal cord injury via Plexin-B2. Nat Neurosci. 2020;23(3):337-50

9. Zeng $\mathrm{H}$, et al. Lentivirus-mediated downregulation of alpha-synuclein reduces neuroinflammation and promotes functional recovery in rats with spinal cord injury. J Neuroinflammation. 2019:16(1):283.

10. Faden $\mathrm{Al}$, et al. Progressive inflammation-mediated neurodegeneration after traumatic brain or spinal cord injury. Br J Pharmacol. 2016;173(4):681-91.

11. Allison DJ, Ditor DS. Immune dysfunction and chronic inflammation following spinal cord injury. Spinal Cord. 2015;53(1):14-8.

12. David S, López-Vales R, Wee Yong V. Harmful and beneficial effects of inflammation after spinal cord injury: potential therapeutic implications. Handb Clin Neurol. 2012;109:485-502.

13. Kong X, Gao J. Macrophage polarization: a key event in the secondary phase of acute spinal cord injury. J Cell Mol Med. 2017;21(5):941-54.

14. Liu G, et al. FK506 attenuates the inflammation in rat spinal cord injury by inhibiting the activation of NF-kappaB in microglia cells. Cell Mol Neurobiol. 2017:37(5):843-55.

15. Norden DM, et al. Bone marrow-derived monocytes drive the inflammatory microenvironment in local and remote regions after thoracic spinal cord injury. J Neurotrauma. 2019;36(6):937-49.

16. Zhou $X, H e X$, Ren Y. Function of microglia and macrophages in secondary damage after spinal cord injury. Neural Regen Res. 2014;9(20):1787-95

17. Orr MB, Gensel JC. Spinal cord injury scarring and inflammation: therapies targeting glial and inflammatory responses. Neurotherapeutics. 2018;15(3):541-53.

18. Chio JCT, et al. The effects of human immunoglobulin $\mathrm{G}$ on enhancing tissue protection and neurobehavioral recovery after traumatic cervical spinal cord injury are mediated through the neurovascular unit. J Neuroinflammation. 2019;16(1):141.

19. Ghosh M, Xu Y, Pearse DD. Cyclic AMP is a key regulator of M1 to M2a phenotypic conversion of microglia in the presence of Th2 cytokines. J Neuroinflammation. 2016;13:9.

20. O'Halloran S, et al. MyD88 acts as an adaptor protein for inflammatory signalling induced by amyloid- $\beta$ in macrophages. Immunol Lett. 2014;162(1 Pt A):109-18

21. Kroner $\mathrm{A}$, et al. TNF and increased intracellular iron alter macrophage polarization to a detrimental M1 phenotype in the injured spinal cord. Neuron. 2014:83(5):1098-116.

22. Ren $\mathrm{H}$, et al. Regulation of inflammatory cytokines for spinal cord injury repair through local delivery of therapeutic agents. Adv Sci (Weinh). 2018;5(11):1800529.
23. Weisser SB, et al. Generation and characterization of murine alternatively activated macrophages. Methods Mol Biol. 2013;946:225-39.

24. Kigerl KA, et al. Identification of two distinct macrophage subsets with divergent effects causing either neurotoxicity or regeneration in the injured mouse spinal cord. J Neurosci. 2009;29(43):13435-44.

25. Mothe AJ, et al. RGMa inhibition with human monoclonal antibodies promotes regeneration, plasticity and repair, and attenuates neuropathic pain after spinal cord injury. Sci Rep. 2017;7(1):10529.

26. Dyck $\mathrm{S}$, et al. Perturbing chondroitin sulfate proteoglycan signaling through LAR and PTPsigma receptors promotes a beneficial inflammatory response following spinal cord injury. J Neuroinflammation. 2018;15(1):90.

27. Gordon S, Martinez FO. Alternative activation of macrophages: mechanism and functions. Immunity. 2010;32(5):593-604.

28. Murray PJ, et al. Macrophage activation and polarization: nomenclature and experimental guidelines. Immunity. 2014;41(1):14-20.

29. Zhang LX, et al. Grafted bone marrow stromal cells: a contributor to glial repair after spinal cord injury. Neuroscientist. 2015;21(3):277-89.

30. Thompson CD, et al. The therapeutic role of interleukin-10 after spinal cord injury. J Neurotrauma. 2013:30(15):1311-24.

31. Hellenbrand DJ, et al. Sustained interleukin-10 delivery reduces inflammation and improves motor function after spinal cord injury. J Neuroinflammation. 2019;16(1):93.

32. Zhang $\mathrm{H}$, et al. Isosteviol sodium protects against ischemic stroke by modulating microglia/macrophage polarization via disruption of GAS5/miR-146a-5p sponge. Sci Rep. 2019;9(1):12221.

33. Wynn TA, Chawla A, Pollard JW. Macrophage biology in development, homeostasis and disease. Nature. 2013;496(7446):445-55.

34. Block ML, Zecca L, Hong JS. Microglia-mediated neurotoxicity: uncovering the molecular mechanisms. Nat Rev Neurosci. 2007;8(1):57-69.

35. Hong LTA, et al. An injectable hydrogel enhances tissue repair after spinal cord injury by promoting extracellular matrix remodeling. Nat Commun. 2017:8(1):533.

36. Varvel NH, et al. Microglial repopulation model reveals a robust homeostatic process for replacing CNS myeloid cells. Proc Natl Acad Sci U S A. 2012;109(44):18150-5.

37. Jia Z, et al. Oxidative stress in spinal cord injury and antioxidantbased intervention. Spinal Cord. 2012:50(4):264-74.

38. Busch SA, Silver J. The role of extracellular matrix in CNS regeneration. Curr Opin Neurobiol. 2007;17(1):120-7.

39. Boato $F$, et al. Absence of IL-1 $\beta$ positively affects neurological outcome, lesion development and axonal plasticity after spinal cord injury. J Neuroinflammation. 2013;10:6.

40. Nigam S, Schewe T. Phospholipase A(2)s and lipid peroxidation. Biochim Biophys Acta. 2000;1488(1-2):167-81.

41. Bao F, et al. Increased oxidative activity in human blood neutrophils and monocytes after spinal cord injury. Exp Neurol. 2009;215(2):308-16.

42. Rathore $\mathrm{Kl}$, et al. Ceruloplasmin protects injured spinal cord from iron-mediated oxidative damage. J Neurosci. 2008;28(48):12736-47.

43. Vergadi $\mathrm{E}$, et al. Akt signaling pathway in macrophage activation and M1/M2 polarization. J Immunol. 2017;198(3):1006-14.

44. Malyshev I, Malyshev Y. Current concept and update of the macrophage plasticity concept: intracellular mechanisms of reprogramming and M3 macrophage "Switch" phenotype. Biomed Res Int. 2015;2015:341308

45. Arranz A, et al. Akt1 and Akt2 protein kinases differentially contribute to macrophage polarization. Proc Natl Acad Sci U S A. 2012;109(24):9517-22.

46. Ruffell D, et al. A CREB-C/EBPbeta cascade induces M2 macrophagespecific gene expression and promotes muscle injury repair. Proc Natl Acad Sci U S A. 2009:106(41):17475-80.

47. Croker BA, Kiu H, Nicholson SE. SOCS regulation of the JAK/STAT signalling pathway. Semin Cell Dev Biol. 2008;19(4):414-22.

48. Xu F, et al. Akt1-mediated regulation of macrophage polarization in a murine model of Staphylococcus aureus pulmonary infection. J Infect Dis. 2013;208(3):528-38.

49. D'Souza B, Miyamoto A, Weinmaster G. The many facets of Notch ligands. Oncogene. 2008;27(38):5148-67. 
50. $\mathrm{Xu} \mathrm{H}$, et al. Notch-RBP-J signaling regulates the transcription factor IRF8 to promote inflammatory macrophage polarization. Nat Immunol. 2012;13(7):642-50.

51. Zhao J, et al. IRF-8/interferon (IFN) consensus sequence-binding protein is involved in Toll-like receptor (TLR) signaling and contributes to the cross-talk between TLR and IFN-gamma signaling pathways. J Biol Chem. 2006;281(15):10073-80.

52. Wang $Y C$, et al. Notch signaling determines the M1 versus M2 polarization of macrophages in antitumor immune responses. Cancer Res. 2010;70(12):4840-9

53. Zhang W, Xu W, Xiong S. Blockade of Notch1 signaling alleviates murine lupus via blunting macrophage activation and $\mathrm{M} 2 \mathrm{~b}$ polarization. J Immunol. 2010;184(11):6465-78.

54. Kono Y, et al. In vitro evaluation of inhibitory effect of nuclear factorkappaB activity by small interfering RNA on pro-tumor characteristics of M2-like macrophages. Biol Pharm Bull. 2014;37(1):137-44.

55. Rawlings JS, Rosler KM, Harrison DA. The JAK/STAT signaling pathway. J Cell Sci. 2004;117(Pt 8):1281-3.

56. Yeh $\mathrm{CH}$, et al. Protective effect of wogonin on proinflammatory cytokine generation via Jak1/3-STAT1/3 pathway in lipopolysaccharide stimulated BV2 microglial cells. Toxicol Ind Health. 2015;31(10):960-6.

57. Satoh T, et al. Critical role of Trib1 in differentiation of tissue-resident M2-like macrophages. Nature. 2013;495(7442):524-8.

58. Eguchi J, et al. Interferon regulatory factor 4 regulates obesity-induced inflammation through regulation of adipose tissue macrophage polarization. Diabetes. 2013;62(10):3394-403.

59. Bhattacharjee $A$, et al. IL-4 and IL-13 employ discrete signaling pathways for target gene expression in alternatively activated monocytes/macrophages. Free Radic Biol Med. 2013:54:1-16.

60. Pello OM, et al. Role of C-MYC in alternative activation of human macrophages and tumor-associated macrophage biology. Blood. 2012;119(2):411-21.

61. Gordon S. Alternative activation of macrophages. Nat Rev Immunol. 2003;3(1):23-35.

62. Qin $\mathrm{H}$, et al. SOCS3 deficiency promotes M1 macrophage polarization and inflammation. J Immunol. 2012;189(7):3439-48.

63. Kamato D, Little PJ. Smad2 linker region phosphorylation is an autonomous cell signalling pathway: implications for multiple disease pathologies. Biomed Pharmacother. 2020;124:109854.

64. Hata A, Chen YG. TGF-beta signaling from receptors to Smads. Cold Spring Harb Perspect Biol. 2016;8(9):a022061.

65. Zhang F, et al. TGF-beta induces M2-like macrophage polarization via SNAIL-mediated suppression of a pro-inflammatory phenotype. Oncotarget. 2016;7(32):52294-306.

66. Li H, et al. Transcriptional regulation of macrophages polarization by MicroRNAs. Front Immunol. 2018:9:1175.

67. Li W, et al. Nobiletin-ameliorated lipopolysaccharide-induced inflammation in acute lung injury by suppression of NF-kappaB pathway in vivo and vitro. Inflammation. 2018:41(3):996-1007.

68. Ye $\mathrm{H}$, et al. Distinct molecular mechanism for initiating TRAF6 signalling. Nature. 2002:418(6896):443-7.

69. Woronicz JD, et al. IkappaB kinase-beta: NF-kappaB activation and complex formation with IkappaB kinase-alpha and NIK. Science. 1997;278(5339):866-9.

70. Porta C, et al. Tolerance and M2 (alternative) macrophage polarization are related processes orchestrated by p50 nuclear factor kappaB. Proc Natl Acad Sci U S A. 2009;106(35):14978-83.

71. Luu K, et al. STAT1 plays a role in TLR signal transduction and inflammatory responses. Immunol Cell Biol. 2014;92(9):761-9.

72. Albu S, et al. Clinical effects of intrathecal administration of expanded Wharton jelly mesenchymal stromal cells in patients with chronic complete spinal cord injury: a randomized controlled study. Cytotherapy. 2020. https://doi.org/10.1016/j.jcyt.2020.08.008.

73. Liau LL, et al. Characteristics and clinical applications of Wharton's jelly-derived mesenchymal stromal cells. Curr Res Transl Med. 2020;68(1):5-16.

74. Wu GH, et al. Recovery of paralyzed limb motor function in canine with complete spinal cord injury following implantation of MSCderived neural network tissue. Biomaterials. 2018:181:15-34.
75. Albayrak O, et al. Mesenchymal stem cell therapy improves erectile dysfunction in experimental spinal cord injury. Int J Impot Res. 2020:32(3):308-16.

76. Wang $L$, et al. Mesenchymal stem cell-derived exosomes reduce A1 astrocytes via downregulation of phosphorylated NFkappaB P65 subunit in spinal cord injury. Cell Physiol Biochem. 2018;50(4):1535-59.

77. Vaquero J, et al. Intrathecal administration of autologous mesenchymal stromal cells for spinal cord injury: safety and efficacy of the 100/3 guideline. Cytotherapy. 2018:20(6):806-19.

78. Lankford KL, et al. Intravenously delivered mesenchymal stem cellderived exosomes target M2-type macrophages in the injured spinal cord. PLoS One. 2018:13(1):e0190358.

79. Morita T, et al. Intravenous infusion of mesenchymal stem cells promotes functional recovery in a model of chronic spinal cord injury. Neuroscience. 2016;335:221-31.

80. Guo M, et al. Enhancement of neural stem cell proliferation in rats with spinal cord injury by a combination of repetitive transcranial magnetic stimulation (rTMS) and human umbilical cord blood mesenchymal stem cells (hUCB-MSCs). Med Sci Monit. 2020;26:e924445.

81. Yasuda N, et al. Intravenous delivery of mesenchymal stem cells protects both white and gray matter in spinal cord ischemia. Brain Res. 2020;1747:147040.

82. Ruzicka J, et al. Anti-inflammatory compound curcumin and mesenchymal stem cells in the treatment of spinal cord injury in rats. Acta Neurobiol Exp. 2018;78(4):358-74.

83. Yousefifard $M$, et al. A combination of mesenchymal stem cells and scaffolds promotes motor functional recovery in spinal cord injury: a systematic review and meta-analysis. Journal of Neurosurgery-Spine. 2020;32(2):269-84.

84. Nakajima $H$, et al. Transplantation of mesenchymal stem cells promotes an alternative pathway of macrophage activation and functional recovery after spinal cord injury. J Neurotrauma. 2012;29(8):1614-25.

85. Zhou HL, et al. Transplantation of human amniotic mesenchymal stem cells promotes functional recovery in a rat model of traumatic spinal cord injury. Neurochem Res. 2016;41(10):2708-18.

86. Badner $\mathrm{A}$, et al. Splenic involvement in umbilical cord matrix-derived mesenchymal stromal cell-mediated effects following traumatic spinal cord injury. J Neuroinflammation. 2018;15(1):219.

87. Hakim R, et al. Syngeneic, in contrast to allogeneic, mesenchymal stem cells have superior therapeutic potential following spinal cord injury. J Neuroimmunol. 2019:328:5-19.

88. Peng $Z$, et al. Promotion of neurological recovery in rat spinal cord injury by mesenchymal stem cells loaded on nerve-guided collagen scaffold through increasing alternatively activated macrophage polarization. J Tissue Eng Regen Med. 2018;12(3):e1725-36.

89. Caron I, et al. A new three dimensional biomimetic hydrogel to deliver factors secreted by human mesenchymal stem cells in spinal cord injury. Biomaterials. 2016;75:135-47.

90. Doherty TM, et al. Modulation of murine macrophage function by IL-13. J Immunol. 1993;151(12):7151-60.

91. Martinez FO, Helming L, Gordon S. Alternative activation of macrophages: an immunologic functional perspective. Annu Rev Immunol. 2009;27:451-83.

92. Hao J, et al. Involvement of JNK signaling in IL4-induced M2 macrophage polarization. Exp Cell Res. 2017;357(2):155-62.

93. Junttila IS. Tuning the cytokine responses: an update on interleukin (IL)-4 and IL-13 receptor complexes. Front Immunol. 2018;9:888.

94. Czimmerer $Z$, et al. The IL-4/STAT6 signaling axis establishes a conserved microRNA signature in human and mouse macrophages regulating cell survival via miR-342-3p. Genome Med. 2016;8(1):63.

95. Van Dyken SJ, Locksley RM. Interleukin-4- and interleukin-13-mediated alternatively activated macrophages: roles in homeostasis and disease. Annu Rev Immunol. 2013;31:317-43.

96. Dooley D, et al. Cell-based delivery of interleukin-13 directs alternative activation of macrophages resulting in improved functional outcome after Sspinal cord injury. Stem Cell Rep. 2016;7(6):1099-115.

97. Bao C, et al. Blockade of interleukin-7 receptor shapes macrophage alternative activation and promotes functional recovery after spinal cord injury. Neuroscience. 2018;371:518-27. 
98. Bao CS, et al. Transplantation of human umbilical cord mesenchymal stem cells promotes functional recovery after spinal cord injury by blocking the expression of IL-7. Eur Rev Med Pharmacol Sci. 2018;22(19):6436-47.

99. Tsumuraya T, et al. Human mesenchymal stem/stromal cells suppress spinal inflammation in mice with contribution of pituitary adenylate cyclase-activating polypeptide (PACAP). J Neuroinflammation. 2015; $12: 35$

100. Zlotnik A, Yoshie O. Chemokines: a new classification system and their role in immunity. Immunity. 2000;12(2):121-7.

101. Deshmane SL, et al. Monocyte chemoattractant protein-1 (MCP-1): an overview. J Interferon Cytokine Res. 2009;29(6):313-26.

102. Deci MB, et al. Modulating macrophage polarization through CCR2 inhibition and multivalent engagement. Mol Pharm. 2018;15(7):2721-31.

103. Sierra-Filardi $E$, et al. CCL2 shapes macrophage polarization by GM-CSF and M-CSF: identification of CCL2/CCR2-dependent gene expression profile. J Immunol. 2014;192(8):3858-67.

104. Shechter $R$, et al. Recruitment of beneficial M2 macrophages to injured spinal cord is orchestrated by remote brain choroid plexus. Immunity. 2013;38(3):555-69.

105. Matsubara K, et al. Secreted ectodomain of sialic acid-binding lg-like lectin-9 and monocyte chemoattractant protein-1 promote recovery after rat spinal cord injury by altering macrophage polarity. J Neurosci. 2015;35(6):2452-64

106. Sodhi A, Biswas SK. Monocyte chemoattractant protein-1-induced activation of p42/44 MAPK and c-Jun in murine peritoneal macrophages: a potential pathway for macrophage activation. J Interferon Cytokine Res. 2002;22(5):517-26.

107. Li Z, et al. Klf4 alleviates lipopolysaccharide-induced inflammation by inducing expression of MCP-1 induced protein 1 to deubiquitinate TRAF6. Cell Physiol Biochem. 2018:47(6):2278-90.

108. Kapoor N, et al. Transcription factors STAT6 and KLF4 implement macrophage polarization via the dual catalytic powers of MCPIP. J Immunol. 2015;194(12):6011-23.

109. Papa S, et al. Mesenchymal stem cells encapsulated into biomimetic hydrogel scaffold gradually release CCL2 chemokine in situ preserving cytoarchitecture and promoting functional recovery in spinal cord injury. J Control Release. 2018;278:49-56.

110. Yagura K, et al. The enhancement of CCL2 and CCL5 by human bone marrow-derived mesenchymal stem/stromal cells might contribute to inflammatory suppression and axonal extension after spinal cord injury. PLoS One. 2020;15(3):e0230080.

111. Marques RE, et al. Targeting CCL5 in inflammation. Expert Opin Ther Targets. 2013;17(12):1439-60.

112. Bronte $V$, Bria E. Interfering with CCL5/CCR5 at the Tumor-Stroma Interface. Cancer Cell. 2016;29(4):437-9.

113. Jaguin M, et al. Polarization profiles of human M-CSF-generated macrophages and comparison of M1-markers in classically activated macrophages from GM-CSF and M-CSF origin. Cell Immunol. 2013;281(1):51-61.

114. Simpson RJ, Jensen SS, Lim JW. Proteomic profiling of exosomes: current perspectives. Proteomics. 2008;8(19):4083-99.

115. Tkach M, Théry C. Communication by extracellular vesicles: where we are and where we need to go. Cell. 2016;164(6):1226-32.

116. Boriachek $\mathrm{K}$, et al. Biological functions and current advances in isolation and detection strategies for exosome nanovesicles. Small. 2018;14(6):1702153.

117. Wortzel l, et al. Exosome-mediated metastasis: communication from a distance. Dev Cell. 2019;49(3):347-60.

118. Zhao J, et al. Mesenchymal stromal cell-derived exosomes attenuate myocardial ischaemia-reperfusion injury through miR-182-regulated macrophage polarization. Cardiovasc Res. 2019;115(7):1205-16.
119. Deng $\mathrm{S}$, et al. Exosomes from adipose-derived mesenchymal stem cells ameliorate cardiac damage after myocardial infarction by activating S1P/SK1/S1PR1 signaling and promoting macrophage M2 polarization. Int J Biochem Cell Biol. 2019;114:105564.

120. Song $Y$, et al. Exosomal miR-146a contributes to the enhanced therapeutic efficacy of interleukin-1 $\beta$-primed mesenchymal stem cells against sepsis. Stem Cells. 2017;35(5):1208-21.

121. $\mathrm{Ni} \mathrm{H}$, et al. Exosomes derived from bone mesenchymal stem cells ameliorate early inflammatory responses following traumatic brain injury. Front Neurosci. 2019;13:14.

122. Zhao $C$, et al. Exosomes derived from bone marrow mesenchymal stem cells inhibit complement activation in rats with spinal cord injury. Drug Des Devel Ther. 2019;13:3693-704

123. Sun $\mathrm{G}$, et al. hucMSC derived exosomes promote functional recovery in spinal cord injury mice via attenuating inflammation. Mater Sci Eng C Mater Biol Appl. 2018;89:194-204.

124. Hu X, et al. Severe hypoxia exerts parallel and cell-specific regulation of gene expression and alternative splicing in human mesenchymal stem cells. BMC Genom. 2014;15:303.

125. Hu X, et al. A\&nbsp; large-scale investigation of hypoxia-preconditioned allogeneic mesenchymal stem cells for myocardial repair in nonhuman primates: paracrine activity without remuscularization. Circ Res. 2016;118(6):970-83.

126. Hu X, et al. Leptin signaling is required for augmented therapeutic properties of mesenchymal stem cells conferred by hypoxia preconditioning. Stem Cells. 2014;32(10):2702-13.

127. Zhilai Z, et al. Preconditioning in lowered oxygen enhances the therapeutic potential of human umbilical mesenchymal stem cells in a rat model of spinal cord injury. Brain Res. 2016;1642:426-35.

128. Liu W, et al. Exosome-shuttled miR-216a-5p from hypoxic preconditioned mesenchymal stem cells repair traumatic spinal cord injury by shifting microglial M1/M2 polarization. J Neuroinflammation. 2020;17(1):47.

129. Shao M, et al. Exosomes from long noncoding RNA-Gm37494-ADSCs repair spinal cord injury via shifting microglial M1/M2 polarization. Inflammation. 2020;43(4):1536-47.

130. Daniel B, et al. The nuclear receptor PPARy controls progressive macrophage polarization as a ligand-insensitive epigenomic ratchet of transcriptional memory. Immunity. 2018;49(4):615-26.e6.

131. Liu W, et al. Exosomes derived from bone mesenchymal stem cells repair traumatic spinal cord injury by suppressing the activation of A1 neurotoxic reactive astrocytes. J Neurotrauma. 2019;36(3):469-84.

132. Lee JR, et al. Targeted delivery of mesenchymal stem cell-derived nanovesicles for spinal cord injury treatment. Int J Mol Sci. 2020;21(11):4185

133. Potas JR, et al. Augmented locomotor recovery after spinal cord injury in the athymic nude rat. J Neurotrauma. 2006:23(5):660-73.

134. Jones TB. Lymphocytes and autoimmunity after spinal cord injury. Exp Neurol. 2014;258:78-90.

135. Kilcoyne $M$, et al. Differential glycosylation expression in injured rat spinal cord treated with immunosuppressive drug cyclosporin-A. ACS Omega. 2019;4(2):3083-97.

136. Putatunda R, Bethea JR, Hu WH. Potential immunotherapies for traumatic brain and spinal cord injury. Chin J Traumatol. 2018;21(3):125-36.

\section{Publisher's note}

Springer Nature remains neutral with regard to jurisdictional claims in published maps and institutional affiliations. 